\title{
Géneros Goniini (Diptera: Tachinidae: Exoristiinae) de Cusco, Perú: clave, redescripciones y distribución
}

\author{
Goniini genera (Diptera: Tachinidae: Exoristiinae) \\ from Cusco, Perú: key, redescriptions and distribution \\ Lizeth Paucar D. ${ }^{1}$, Christian R. González ${ }^{2}$, Erick Yábar L. ${ }^{*}$
}

\begin{abstract}
RESUMEN
Tachinidae es una de las familias de Diptera más diversificadas, y la más grande de Oestroidea, con más de 8.500 especies descritas en más de 1.500 géneros y distribuidas en todas las regiones zoogeográficas del planeta. Se reportan para Cusco seis géneros de Goniini (Diptera, Tachinidae): Araucosimus Aldrich, Chaetocnephalia Townsend, Chaetocraniopsis Townsend, Dolichocnephalia Townsend, Germariopsis Townsend y Gonia Meigen. Los géneros Chaetocnephalia, Chaetocraniopsis, Dolichocnephalia y Germariopsis se reportan por primera vez para Cusco y Araucosimus por primera vez para Perú. Se incluye una clave para los seis géneros estudiados.
\end{abstract}

Palabras claves: taxonomía, Goniini, claves, Región Neotropical, Perú.

\section{ABSTRACT}

The family Tachinidae is one of the most diverse of all the insect families with more than 8,500 described species classified into more than 1,500 genera. Six genera of Goniini (Diptera, Tachinidae) are reported to Cusco: Araucosimus Aldrich, Chaetocnephalia Townsend, Chaetocraniopsis Townsend, Dolichocnephalia Townsend, Germariopsis Townsend y Gonia Meigen. The genus Araucosimus is cited for the first time to Peru. Genera Chaetocnephalia, Chaetocraniopsis, Dolichocnephalia, and Germariopsis are cited for the first time to Cusco. A key to the six genera is included.

Key words: taxonomy, Goniini, keys, Neotropical Region, Perú.

\section{Introducción}

Los Tachinidae son un grupo de muscoideos caliptrados de la superfamilia Oestroidea, con más de 8.500 especies clasificadas en un número superior a los 1.500 géneros y distribuidas en todas las regiones zoogeográficas del planeta (O'Hara, 2013). Actualmente, la familia se subdivide en 4 subfamilias: Exoristinae, Dexiinae, Phasiinae y Tachininae, aunque no existe certeza de la monofilia de éstas (Wood \& Zumbado, 2010). La gran mayoría de sus especies son parasitoides de insectos y otros artrópodos (Gramajo et al., 2013), aunque existen algunas especies que no causan la muerte a sus hospederos (DeVries, 1984). Las especies de Tachinidae se encuentran representados en todos los ambientes terrestres incluyendo desiertos, bosques, pasturas, montañas y tundra (Stireman et al., 2006).

En la Región Neotropical, la subfamilia Exoristinae, Goniinae sensu Guimaraes (1971), comprende 21 tribus, una de las cuales es Goniini. Guimaraes (1971) divide a los Goniini en dos subtribus: Goniina (con 34 géneros y 49 especies) y Chaetogaediina (con 7 géneros y 23 especies). La subtribu Goniina reúne 11 géneros citados para Perú: Chaetocnephalia Townsend, Cnephalodes Townsend, Dolichocnephalia Townsend, Dolichogonia Townsend, Germariopsis Townsend, Gonia Meigen, Olenochaeta Townsend, Peracroglossa Townsend, Perumyia Arnaud, Phasiatacta Townsend y Protogoniops Townsend; mientras que la subtribu Chaetogaediina cita solamente al género Gaediopsis Brauer \& Bergenstamm (Guimaraes, 1971).

\footnotetext{
Universidad Nacional de San Antonio Abad del Cusco, Cusco, Perú.

2 Instituto de Entomología, Universidad Metropolitana de Ciencias de la Educación, Santiago, Chile.

* Autor por correspondencia: erick.yabar@unsaac.edu.pe
} 
Existen diversos estudios que incluyen a las especies de Goniini en el cono sur de América del Sur, aunque solo marginalmente consideran la zona en la cual está centrado este estudio. Townsend (1912) estudió los géneros de Goniini, los cuales fueron inicialmente tratados dentro de la subfamilia Salmaciinae (Familia Hystriciidae), incluyendo varios géneros de distribución peruana: Phasiatacta Townsend, Cnephalodopsis Townsend, Salmacia Meigen, Protogonia Townsend, Triachora Townsend y Belvosia Robineau-Desvoidy. Townsend (1915), al tratar la subfamilia Salmaciinae como familia, menciona 3 géneros para Perú: Plagimasicera Townsend, Protogoniopsis Townsend y Echinomasicera Townsend. Townsend (1928) describe un nuevo género y especie de la costa de Perú y Chile. Aldrich (1934), en su tratamiento de la fauna de Tachinidae de la Patogonia y el sur de Chile, describe nuevas especies y redescribe especies tratadas por otros autores. Townsend (1936) define y caracteriza la tribu Goniini, además de ofrecer una clave para reconocer Townsend (1941) incluye, en la tribu Goniini, 62 géneros, de los cuales 7 presentan distribución peruana: Chaetocnephalia Townsend, Cnepahlodes Townsend, Dolichocnephalia Townsend, Dolichogonia Townsend, Germariopsis Townsend, Peracroglossa Townsend y Phasiatacta Townsend. Cortés (1944) estudió tres géneros goniinos: Chaetocraniopsis Townsend, Chaetocnephalia Townsend y Araucosimus Aldrich, considerando, principalmente, la presencia de setas en el parafacial y la presencia, o no, de setas apicales escutelares. Cortés \& Campos (1970), al estudiar los Tachinidae del extremo norte de Chile, entregan claves para diferenciar los géneros además de entregar nuevos antecedentes distribucionales de las especies incluidas. Cortés (1986) caracterizó la subfamilia Exoristinae, para las especies australes de Argentina y Chile, aunque la diagnosis es válida para separar los géneros de Exoristinae presentes en Cusco. Posteriormente, González \& Vergés (2004), revisaron las especies de Goniini de distribución chilena citando 10 géneros: Enchomyia Aldrich, Philocorus de distribución chilena Cortés, Coscaronia Cortés, Dolichocnephalia Townsend, Chaetocraniopsis Townsend, Protogoniops Townsend, Araucogonia Cortés, y Chaetocnephalia Townsend.

Pese a los esfuerzos mencionados, y dada la importancia que los Tachinidae presentan como parasitoides de algunas plagas, polinizadores y otros servicios ecosistémicos, existe casi un completo desconocimiento de la entomofauna de taquínidos para el área del Cusco.

El objetivo del presente trabajo es reportar los géneros goniinos presentes en Cusco (Perú), proponer una clave para su identificación así como entregar datos de su distribución geográfica en la zona.

\section{Material y Métodos}

\section{Área de estudio}

El área de estudio se ubica entre 1.084 a $3.813 \mathrm{msnm}$ (Figura 1) y presenta una fauna representativa caracterizada por: Notiobia (Coleoptera, Carabidae), Listroderes, Macrostyphlus, Rhigopsidius y Trichocyphus (Coleoptera, Curculionidae), Nicrophorus (Coleoptera, Silphidae), Pompilocalus (Hymenoptera, Pompilidae), Chlorion (Hymenoptera, Sphecidae), Hemerobius (Neuroptera, Hemerobiidae) y otros (Morrone, 2001). En forma general la vegetación está compuesta por efedras y angiospermas (Morrone, 2001). Se observan pastizales y se cultiva papa, maíz, habas, quinua, etc. generalmente en terrenos de secano. Se presentan dos épocas climáticas bien marcadas: lluvias (noviembre-marzo) y secas (abril-octubre). Comprende las provincias de Canchis, Cusco, Chumbivilcas, Paucartambo, Quispicanchi y Urubamba.

\section{Especímenes}

Se examinaron 62 ejemplares del material depositado en la Colección Entomológica de la Universidad Nacional de San Antonio Abad del Cusco (CEUC-UNSAAC) (Tabla 1), además de colectas adicionales en el área de estudio mediante red entomológica. Las colecciones no siguieron un muestreo sistemático, realizándose al azar por diferentes personas, tal como figura en las etiquetas del material estudiado. Los ejemplares capturados fueron muertos en cámara letal (KCN) y llevados al laboratorio donde fueron montados con alfileres entomológicos y etiquetados. Los datos de las etiquetas consignan información sobre localidad, altitud, coordenadas, fecha y nombre del colector. Todas las colecciones fueron hechas durante el día debido a que los Tachinidae, 


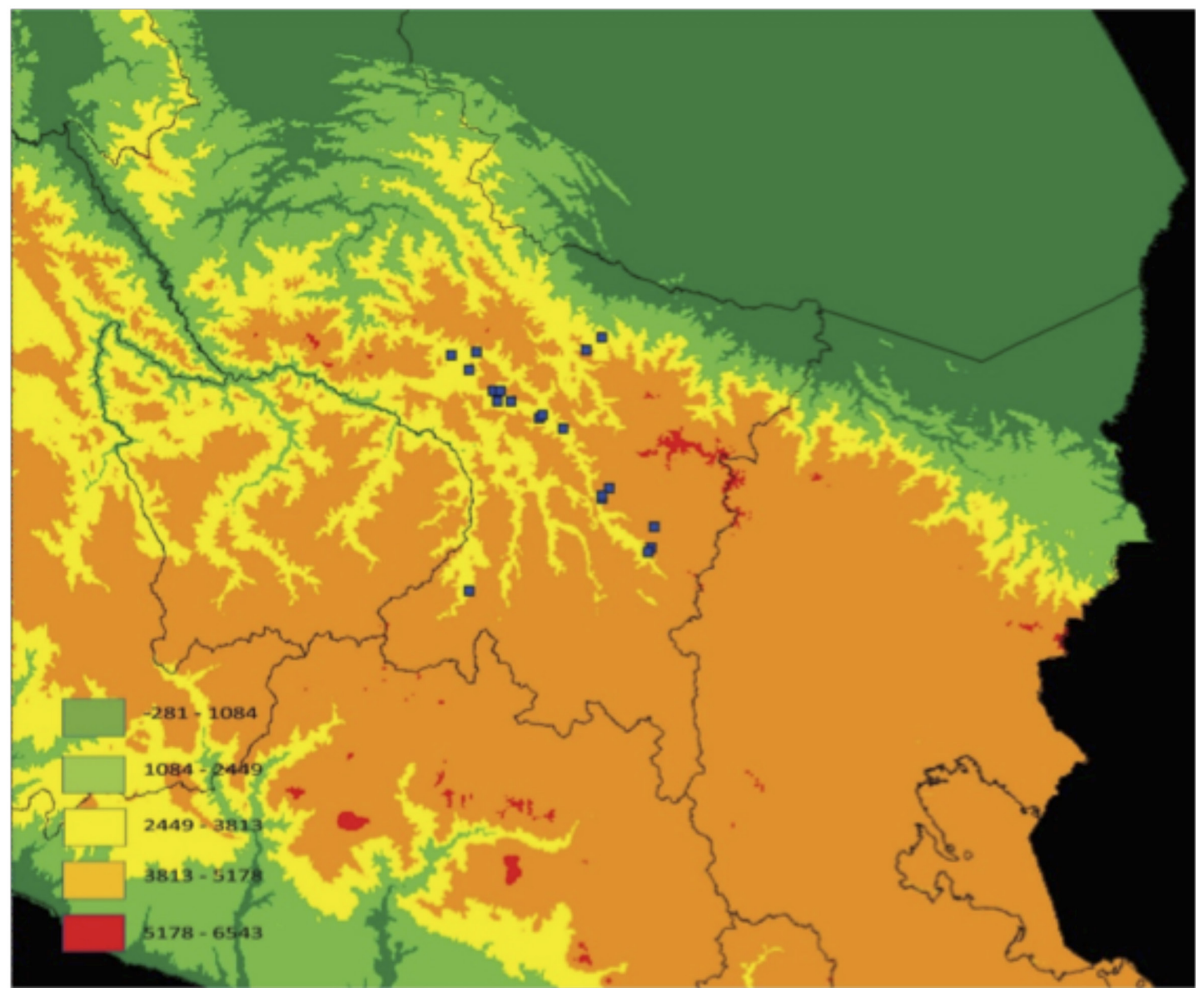

Figura 1. Mapa de ubicación de los géneros de Goniini estudiados en Cusco.

Tabla 1. Número de ejemplares estudiados por género.

\begin{tabular}{lc}
\hline \multicolumn{1}{c}{ Género } & Número de ejemplares \\
\hline Araucosimus & 4 \\
Chaetocnephalia & 9 \\
Chaetocraniopsis & 3 \\
Dolichocnephalia & 3 \\
Germariopsis & 1 \\
Gonia & 42 \\
\hline
\end{tabular}

en general, son voladores diurnos. Como no se siguió un muestreo sistemático las colecciones fueron hechas en campos de cultivos diversos y áreas no cultivadas. Todo el material queda depositado en la Colección Entomológica de la Universidad Nacional de San Antonio Abad del Cusco (CEUC-UNSAAC).

La determinación del material se realizó en los laboratorios de Entomología de la UNSAAC y del Instituto de Entomología de la Universidad Metropolitana de Ciencias de la Educación de Santiago (UMCE) como parte de la tesis elaborada por la primera de los autores. Las observaciones fueron realizadas empleando un microscopio estereoscópico CarlZeiss Discovery V20 y con las claves de Aldrich (1934), Cortés (1944, 1986), Cortés y Campos (1970), González y Vergés (2004) y Townsend $(1912,1913,1915)$. Para la diagnosis morfológica de los géneros se siguió la terminología propuesta por Cumming \& Wood (2009). Incluso tratándose de un estudio a nivel genérico las descripciones son lo más amplias posible, en el entendido que más adelante se harán las determinaciones a nivel específico. Esto debido a que, por el momento, no nos fue posible llegar al nivel de especie por cuanto algunas descripciones originales son muy escuetas y, especialmente, porque no contamos con una colección de referencia que nos hubiera 
permitido realizar determinaciones más precisas por comparación. Para la descripción de los géneros éstos se ordenan en orden alfabético, independientemente del orden que ocupan en la clave, ya que ésta se basa en caracteres diagnósticos y no sigue un orden preestablecido. Las medidas fueron tomadas empleando un ocular micrométrico; la longitud se refiere a la distancia entre la frente y el extremo del abdomen. No se ha discriminado entre sexos debido, fundamentalmente, a que algunos ejemplares no se encontraban en buenas condiciones (por ejemplo, sin abdomen) y, en otros casos, no era muy clara la separación entre sexos; de este modo, para cada género se registra la longitud promedio y la desviación estándar, excepto en el caso del ejemplar de Germariopsis, del cual se extrajo el abdomen para la preparación de genitalias. Las fotografías fueron tomadas con cámara digital Axiocam ICc 5 adosada a un microscopio estereoscópico Zeiss Discovery V20.

\section{Resultados}

Producto del estudio del material, se determinaron seis géneros, todos ellos prácticamente nuevos para Cusco.

\section{Clave para los géneros Goniini (Diptera, Tachinidae) del Cusco}

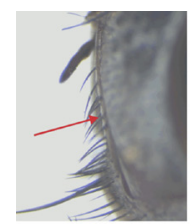

A
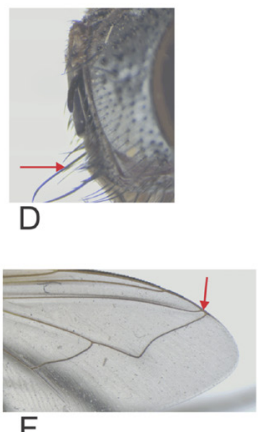

F

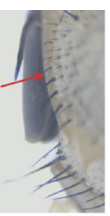

$\mathrm{B}$



C


Figura 2. A: sutura fronto-genal setulosa (Chaetocraniopsis); B: sutura fronto-genal no setulosa (Gonia); C: frontoclipeo muy desarrollado (Dolichocnephalia); D: frontoclipeo no desarrollado (Chaetocraniopsis); E: $\mathrm{R}_{4+5}$ con corto peciolo (Germariopsis); F: $\mathrm{R}_{4+5}$ sin peciolo (Gonia); G: Base de $\mathrm{R}_{4+5}$ setulosa casi hasta r-m (Gonia); $\mathrm{H}: \mathrm{R}_{4+5}$ con setas pequeñas (Araucosimus); I: apicales escutelares piliformes o ausentes (Araucosimus); J: apicales escutelares espiniformes, erectas (Chaetocnephalia).

1. Sutura fronto-genal setulosa (Figura 2, A)

Sutura fronto-genal no setulosa (Figura 2, B)

2. Frontoclipeo muy desarrollado; perfil facial sobrepasa al perfil frontal (Figura 2, C

Dolichocnephalia Townsend

Frontoclipeo no desarrollado; perfil frontal más pronunciado que el perfil facial (Figura 2, D)......

Chaetocraniopsis Townsend

3. Celda $\mathrm{r}_{4+5}$ con un corto peciolo (Figura 2, E).

Germariopsis Townsend

Celda $\mathrm{r}_{4+5}$ sin peciolo, abierta o cerrada exactamente en el margen alar (Figura 2, F)

4. Base de la vena $\mathrm{R}_{4+5}$ setulosa casi hasta r-m (Figura 2, G)

Gonia Meigen

Base de la vena $\mathrm{R}_{4+5}$ con $4-5$ con sétulas pequeñas (Figura 2, H)

5. Setas apicales escutelares piliformes o ausentes (Figura 2, I)

Araucosimus Aldrich

Setas apicales escutelares espiniformes, erectas, más cortas que las laterales (Figura 2, J) 
Araucosimus Aldrich, 1934. (Figura 3, A: hábito)

Especie tipo: A. bullocki Aldrich (designación original).

Referencias. Aldrich, 1934: 88 (descripción género); Townsend, 1941: 10 (redescripción); Cortés y Campos, 1970: 76 (distribución); Guimaraes, 1971: 173 (catálogo); Cortés \& Campos, 1974: 113 (clave géneros); González y Vergés, 2004: 43 (clave especies).

\section{Diagnosis}

Longitud 10,83 $\pm 0,29(n=4)$. Cabeza dolicocéfala; ojos desnudos, setas frontales en tres hileras, sutura fronto-genal sin setas; pedicelo café amarillento, post-pedicelo negro, apenas más largo que el pedicelo; Palpos muy largos, casi alcanzando el ángulo fronto-clipeal, café. Tórax gris con 3 bandas de pruina blanquecina centrales y dos bandas blanquecinas laterales entre el lóbulo postpronotal, parte superior de la notopleura y el área supra alar; celda $\mathrm{r}_{4+5}$ cerrada exactamente en el margen alar; escutelo amarillo; abdomen negro con cuatro hileras paralelas de manchas triangulares de pruina blanquecina, dos centrales y dos laterales en los tergitos 2 a 4 , tergito 5 amarillo; adicionalmente el tergito 2 cubierto con pruina blanquecina visible solo en cierto ángulo.

\section{Descripción}

Cabeza. Dolicocéfala, casi enteramente recubierta de pruina plateada; ojos desnudos, frente café, casi del mismo ancho en toda su longitud; dos manchas oscuras entre los ojos y el triángulo ocelar; área interfrontal café claro, triángulo ocelar con setas cortas, erectas, setas frontales en tres hileras con 6-7 setas, las dos externas irregulares; la seta frontal más baja llega hasta el extremo del pedicelo; 2 setas orbitales proclinadas en las hembras, ninguna en el macho; parafacial tan ancho como el ancho del ojo, con 3 hileras irregulares de setas; en cierto ángulo el parafacial adquiere un tono café oscuro; impresión transversa debajo del parafacial café rojizo; sutura fronto-genal sin setas; palpos maxilares largos, casi hasta el borde fronto-clipeal, amarillos, suavemente engrosados hacia el ápice, antenas con el escapo corto, café amarillento, pedicelo café amarillento, postpedicelo negro, apenas más largo que el pedicelo

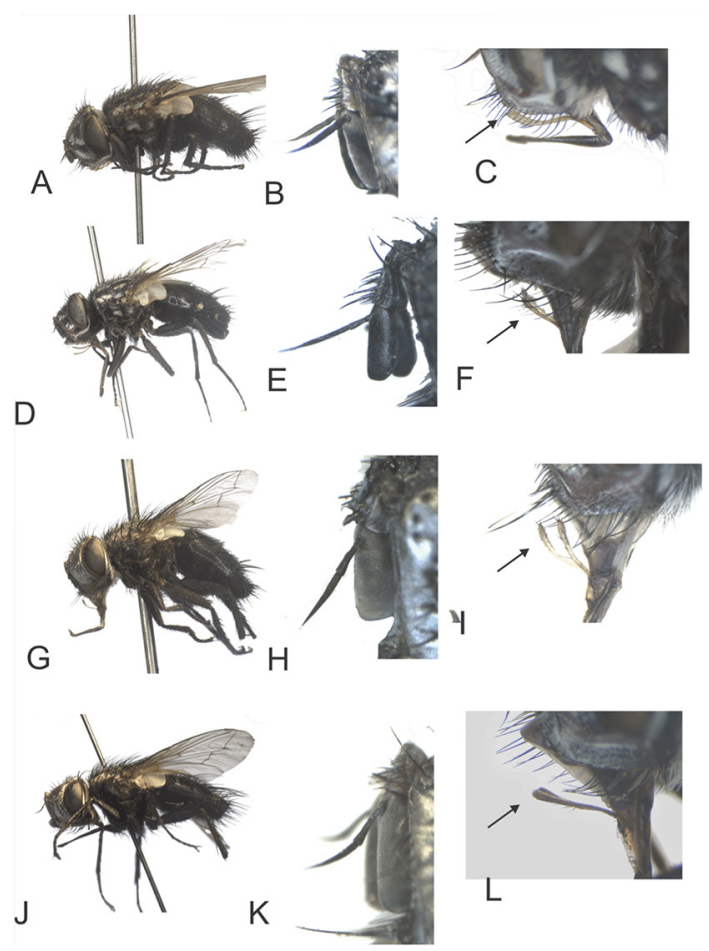

Figura 3. A Araucosimus, hábito; B: Araucosimus, antena; C: Araucosimus, palpo; D: Chaetocnephalia, hábito; E: Chaetocnephalia, antena; F: Chaetocnephalia, palpo; G: Chaetocraniopsis, hábito; H: Chaetocraniopsis, antena; I; Chaetocraniopsis, palpo; J: Dolichocnephalia, hábito; K: Dolichocnephalia, antena; L, Dolichocnephalia, palpo.

(Figura 3, B). Arista desnuda, aristómero 1 corto, aristómero 3 algo más de 3 veces la longitud del 2; gena con pruina blanquecina. Palpos muy largos, casi alcanzan el ángulo fronto-clipeal, café, casi del mismo grosor, margen inferior con setas finas en el tercio medio y casi el tercio apical, margen superior con unas pocas setas cortas en el tercio basal, ápice con unas pocas setas muy cortas (Figura 3, C).

Tórax. Scutum gris con 3 bandas de pruina blanquecina centrales y dos bandas blanquecinas laterales entre el lóbulo post-pronotal, parte superior de la notopleura y el área supra alar. Postpronoto con 3 setas en línea; Scutum: acrosticales 3:3, dorsocentrales $4: 4$, intraalares $3: 3$, post alares 2, la posterior más desarrollada, supraalares 3 . Proesterno setoso. Katepisterno con 4 setas, 3 en una hilera superior, la posterior más desarrollada y 1 inferior. Escutelo amarillento, 1 par de discales, 2 pares de basales, 1 par de subapicales y apicales piliformes o ausentes. Alas con venas amarillentas; 
celda $\mathrm{r}_{4+5}$ cerrada exactamente en el margen alar; $\mathrm{R}_{4+5}$ con 3 setas finas en la base; $\mathrm{M}$ formando un ángulo recto y terminando antes del ápice del ala; sección apical de $\mathrm{CuA}_{1}$ menos de la mitad de la sección precedente. Calíptras blancas con el borde ligeramente oscurecido.

Abdomen. Negro con cuatro hileras paralelas de manchas triangulares de pruina blanquecina, dos centrales y dos laterales en los tergitos 2 a 4; tergitos 2 y 3 con un par de setas marginales y 1 par de marginales laterales, tergito 4 con una hilera marginal de setas y setas discales más pequeñas, tergito 5 amarillento con una hilera marginal de setas bien desarrolladas.

Material examinado. 1 $\hat{0}$, Kayra, Cusco, $3.243 \mathrm{~m},-13^{\circ} 33^{\prime},-71^{\circ} 52^{\prime}, 15-2-2001$, E. Yábar col.; 10ิ, Tancarpata, Cusco, 3.394 m, -13³3', -71 ${ }^{\circ} 56^{\prime}, 14-05-2002$, A. Bustamante; $1^{\dagger}{ }^{\top}$, Perayoc, Cusco, Cusco, 3.354 m, -13³1', -7157', 18-021999, E. Yábar; 19, Huayoccari, Urubamba, Cusco, $2.983 \mathrm{~m},-13^{\circ} 19^{\prime},-72^{\circ} 02^{\prime}, 28-07-1998$, R. Aymituma.

Bionomía. En Cusco, este género se encuentra entre 2.983 y $3.394 \mathrm{msnm}$. Ha sido colectado en ambientes con vegetación natural arbustiva. No se tienen registros de hospederos. El rango latitudinal del género abarca desde Angol (Chile): 3748'S que sería el punto más al sur y su rango altitudinal va desde $23 \mathrm{msnm}$ (Valparaíso, Chile) hasta los $3.394 \mathrm{msnm}$ que vendría a ser el punto más alto para el género (Cortés \& Campos, 1974; González \& Vergés, 2004).

Hospederos. Desconocidos.

Comentarios. Se reconoce por las setas parafaciales ubicadas en la región media, escutelo sin setas apicales, las manchas oscuras ubicadas entre los ojos y el triángulo ocelar. Constituye el primer registro para Perú.

Chaetocnephalia Townsend, 1915. (Figura 3, D: hábito)

Especie tipo: Chaetocnephalia alpina Townsend (designación original).

Referencias. Townsend, 1915: 63 (descripción género); Aldrich, 1934: 89 (descripción especie); Townsend, 1941: 13 (redescripción); Cortés, 1944: 120 (descripción especie); Cortés y Campos, 1970: 76 (nueva especie); Guimaraes, 1971: 174 (catálogo); Cortés \& Campos, 1974: 113 (clave géneros); Cortés \& Hichins, 1979: 112 (distribución); Cortés, 1986: 155 (distribución); González y Vergés, 2004: 45 (clave especies).

\section{Diagnosis}

Longitud 10,73 $\pm 0,93$ ( $\mathrm{n}=9$ ); cabeza dolicocéfala, ojos desnudos. Antenas cortas, negras, post-pedicelo apenas más largo que el pedicelo. Palpos largos, sin alcanzar el ángulo vibrisal, amarillos. Scutum con tres bandas de pruina blanquecina, las laterales más tenues pasando la sutura; escutelo café claro; celda $\mathrm{r}_{4+5}$ abierta o cerrada exactamente en el margen alar. Abdomen negro con manchas pequeñas de pruina blanquecina-amarillenta en dos hileras centrales en los tergitos 2 a 5 y dos hileras laterales con las mismas características.

Cabeza. Dolicocéfala, ojos desnudos; sutura fronto-genal sin setas, parafacial casi tan ancho como la mitad del ancho del ojo, oscuro con pruina plateada dispersa, dos hileras irregulares de cerdas casi tan fuertes como las frontales; vitta frontal oscura, algo más ancha hacia el triángulo ocelar; dos hileras de cerdas frontales orientadas hacia adentro; placa fronto-orbital oscura con los márgenes interno y externo con pruina plateada, 1-2 pares de orbitales reclinadas fuertes; antenas cortas, negras, post-pedicelo apenas más largo que el pedicelo, relativamente rectangular con los ángulos redondeados; arista desnuda, aristómero 3 más del doble que el 2 (Figura 3, E); Palpos largos, sin alcanzar el ángulo vibrisal, amarillos, ligeramente engrosados apicalmente, margen inferior con 2-3 setas delgadas en el tercio medio, 3 setas más cortas en el margen superior, tercio apical con 6 o más setas fuertes (Figura 3, F).

Tórax. Negro con tres bandas de pruina blanquecina, las laterales más tenues pasando la sutura; una banda blanquecina entre el lóbulo postpronotal, parte superior de la notopleura y el área supra alar; manchas pequeñas de pruina blanquecina en la parte inferior del anepisterno, anepimeron y apenas visible en el katepisterno; Postpronoto con 3 setas en línea; Scuto: acrosticales 2: 3, dorsocentrales 3: 4 , intraalares $1: 3$, post alares 2 , la posterior más desarrollada, supraalares 1: 3; Prosterno setoso. Katepisterno con 4 setas, 3 en una hilera superior, la posterior más desarrollada y 1 inferior. Escutelo amarillento, 1 par de discales divergentes, 1 par de basales, 1 par de subapicales y apicales espiniformes, erectas y más cortas que las laterales. Alas hialinas 
con venas amarillentas; celda $_{4+5}$ cerrada exactamente en el margen alar; $\mathrm{R}_{4+5}$ con 3 sétulas en la base, $\mathrm{M}$ formando un ángulo recto, no sinuosa, termina antes del ápice alar, porción apical de $\mathrm{CuA}_{1}$ casi la mitad de la longitud de la sección precedente. Calíptras blancas con borde amarillento.

Abdomen. Negro con manchas pequeñas de pruina blanquecina-amarillenta en dos hileras centrales en los tergitos 2 a 5 y dos hileras laterales con las mismas características; tergito $1+2$ sin setas marginales medianas y 1 par de setas marginales laterales, tergito 3 con 1 par de setas marginales centrales y 1 par de marginales laterales, tergito 4 con una hilera de seta marginales, tergito 5 amarillo, sin setas, con 1 hilera de setas marginales.

Material Examinado. 1 $\delta^{\lambda}$, Buena vista, Cusco, 3.471 m, -13³0', -7157', 12-11-1994, E. Yábar; 1 ㅇ, Buena vista, Cusco, $3.471 \mathrm{~m},-13^{\circ} 30^{\prime},-71^{\circ} 57^{\prime}$, 12-11-1994, E. Yábar; 2ð, Pumamarca, Cusco, 3.452 m, -13³0', -7155', 15-11-2008, L. Paucar; 20 , Lucre, Quispicanchis, Cusco, $3.174 \mathrm{~m},-13^{\circ} 38^{\prime}$,

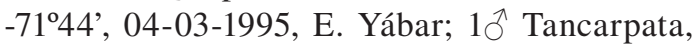
Cusco, 3.394 m, -13³3', -7156' 14-04-2002, A.

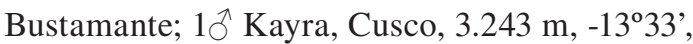

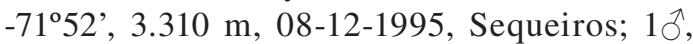
Pitumarca, Canchis, Cusco, 3.576 m, -13 $3^{\circ} 58^{\prime}$, -71²24',17-12-1998, E. Yábar.

Bionomía. La especie Chaetocnephalia andina fue descrita por Townsend (1915) con material colectado en La Oroya (Junín, Perú): $11^{\circ} 31^{\prime} \mathrm{S}, 3.745 \mathrm{msnm}$ y registrada en la localidad de Chapiquiña (Chile): $18^{\circ}$ 23'S 3.577 msnm (Cortés $\&$ Hichins, 1979), puntos que marcarían el rango latitudinal y altitudinal del género. Para Cusco se registra entre 3.174 y $3.576 \mathrm{msnm}$. Townsend (1915) menciona "vegetación corta" por lo que puede asumirse que la especie conocida se encuentra en ambientes de vegetación arbustiva, típica de las alturas citadas.

Hospederos. Desconocidos.

Comentario. Se reconoce por las tres bandas con pruina blanquecina y la banda blanca a los lados del tórax, frontoclipeo muy desarrollado, oscuro y más largo que el perfil frontal. Constituye el primer registro para Cusco.

Chaetocraniopsis Townsend, 1915

(Figura 3, G: hábito)

Valpogonia Townsend, 1928: 163. Especie tipo: C. chilensis (designación original)
Especie tipo: Chaetocraniopsis chilensis Townsend (designación original).

Referencias. Townsend, 1915: 68 (descripción); Townsend, 1928: 163 (descripción género y especie); Townsend, 1941: 14 (redescripción); Cortés, 1944: 116 (clave especies); Cortés y Campos, 1970: 80 (distribución); Guimaraes, 1971: 174 (catálogo); Cortés \& Campos, 1974: 113 (clave géneros); Cortés, 1986: 155 (distribución); González y Vergés, 2004: 49 (clave especies).

\section{Diagnosis}

Longitud: 8,33 $\pm 0,58(\mathrm{n}=3)$; Café oscuro a negruzco; cabeza normal, no dolicocéfala, ojos desnudos; antenas cortas, negras; Palpos largos, casi tan largos como el borde genal inferior. Scutum con tres bandas de pruina gris, anchas, casi unidas más allá de la sutura, lóbulo postpronotal y notopleura cubiertos de pruina gris, dándole al tórax una apariencia cenicienta; pleuras café claro y café oscuro entremezclado; celda $\mathrm{r}_{4+5}$ abierta. Abdomen negro, con cerdas marginales; tergitos 3 y 4 cubiertos con polen blanquecino.

Cabeza. No dolicocéfala, normal, ojos desnudos, sutura fronto-genal con setas casi hasta la base del postpedicelo, frontoclipeo no prominente, perfil frontal no desarrollado, inclinado hasta casi el tercio superior del ojo, más pronunciado que el perfil facial; parafacial casi la mitad del ancho del ojo; parafacial y parafrontal plateadas con polen gris; vitta frontal café claro, de grosor casi uniforme; facialia con una hilera de setas casi hasta la base de las antenas, parafacial casi enteramente cubierta de setas cortas; dos hileras de cerdas frontales inclinadas hacia adentro y una tercera hilera de setas orbitales más débiles, sin fronto-orbitales; sutura fronto-genal setulosa; antenas cortas, negras, post-pedicelo más del doble que el 2; arista delgada, desnuda, aristómero 3 casi 1,5 veces la longitud del aristómero 2 (Figura 3, H); genas café rojizo con ligero polen plateado. Palpos largos, casi tan largos como el borde genal inferior, amarillos, ligeramente engrosados apicalmente, margen inferior con 2 setas en el tercio medio, sin setas en el margen superior, tercio apical con un grupo de setas cortas, dispersas (Figura 3, I).

Tórax. Scutum con tres bandas de pruina gris, anchas, casi unidas más allá de la sutura, lóbulo postpronotal y notopleura cubiertos de pruina gris. Postpronoto con 3 setas en línea; Scutum: 
acrosticales 3: 4, Dorsocentrales 3: 4, intraalares 2: 3 , post alares 2, la posterior más desarrollada, supraalares 1: 3. Prosterno setoso. Katepisterno con 3 setas. Escutelo amarillento, 1 par de discales paralelas, 1 par de basales, 1 par de subapicales y 1 par de apicales divergentes. Alas con venas amarillentas; $\mathrm{R}_{4+5}$ con 4 sétulas largas en la base; celda $r_{4+5}$ abierta, $M$ formando un ángulo recto, poco sinuosa, termina antes del ápice alar; porción distal de $\mathrm{CuA}_{1}$ casi la mitad de la sección precedente. Calíptras totalmente blancas.

Abdomen. Negro, tergito 1+2 con 1 par de marginales centrales y 2 pares de marginales laterales, tergito 3 con 1 par de marginales centrales y 3 pares de marginales laterales, tergito 4 con una hilera de setas marginales, tergito 5 negro, con 2 hileras de setas marginales y varias discales.

Material examinado. $1 \hat{\delta}$, Kayra, Cusco, 3.310 m, -13³3', -71º52', 16-02-1992, E. Yábar; 10 Chiara, Canchis, Cusco, $3.480 \mathrm{~m},-14^{\circ} 15^{\prime},-71^{\circ} 12^{\prime}$, 7-8-1994, E. Yábar; $1{ }^{\widehat{\jmath}}$ Santa Bárbara, Canchis, $4.021 \mathrm{~m},-14^{\circ} 09^{\prime},-71^{\circ} 11^{\prime}, 07-08-1994$, E. Yábar.

Bionomía. La especie Chaetocraniopsis chilensis Townsend fue descrita por Townsend (1915) indicando "Chile", sin más datos; Vergara \& Raven (1989) mencionan esta especie procedente de Chile: Quebrada Escobar, depositada en el Museo de Entomología de la Universidad Nacional Agraria La Molina. Los datos para esta localidad son $71^{\circ} 19^{\prime} \mathrm{O}$ y $33^{\circ} 03^{\prime} \mathrm{S}, 202 \mathrm{msnm}$ que, asumimos, deberían ser los datos de ubicación geográfica de la especie. En Cusco, esta especie se registra entre 3.310 y $4.021 \mathrm{msnm}$ y entre las coordenadas $13^{\circ} 33^{\prime} \mathrm{S}$ y $14^{\circ} 09^{\prime} \mathrm{O}$ lo que amplía grandemente tanto el rango latitudinal como altitudinal. Así, el nuevo rango altitudinal ubica la especie entre los 202 y $4.021 \mathrm{msnm}$. Independientemente de los niveles en los cuales se han colectado los ejemplares estudiados, puede asumirse, también, un amplio rango de hospederos, aunque estos no hayan sido estudiados hasta el momento. La vegetación típica para las zonas de colección en Cusco está formada por arbustos pero, es necesario citar, cultivos como papa, habas, quinua, etc. Puede inferirse, en forma preliminar, que esta especie podría formar parte de un complejo de parasitoides que atacan a varias especies de plagas, especialmente lepidópteros.

Hospederos. Desconocidos.

Comentarios. Se reconoce por las aristas cortas, gruesas, engrosadas uniformemente hacia el ápice, facialia setosa casi hasta la base de las antenas y la curvatura de $\mathrm{M}_{1}$. Es el primer registro para el Cusco.

\section{Dolichocnephalia Townsend, 1915}

(Figura 3, J: Hábito)

Especie tipo: Dolichocnephalia puna Townsend (designación original).

Referencias. Townsend, 1915: 64 (descripción género); Townsend, 1941: 21 (redescripción); Cortés y Campos, 1970: 80 (distribución); Cortés \& Campos, 1974: 114 (clave géneros); Guimaraes, 1971: 174 (catálogo); Cortés \& Hichins, 1979: 112 (distribución); González y Vergés, 2004: 53 (redescripción).

\section{Diagnosis}

Longitud 8,75 $\pm 0,35(n=3)$; Cabeza dolicocéfala, palpos cortos, no alcanzan el ángulo vibrisal, café amarillentos. Scutum grisáceo con 5 bandas de pruina blanquecina, la central más notoria y completa, llega hasta el escutelo; escutelo café amarillento con la base gris; un par de apicales, dos pares de discales y tres pares de laterales, todas más fuertes que las apicales; celda $\mathrm{r}_{4+5}$ abierta; abdomen negro, tergitos 3 y 4 sin discales, con dos bandas de pruina blanquecina en la línea media y dos laterales, tergito 5 casi enteramente recubierto con pruina blanco amarillenta.

Cabeza. Dolicocéfala; ojos desnudos. Perfil frontal casi paralelo con el perfil facial, éste, algo más largo que el perfil frontal; vitta frontal negruzca, de grosor uniforme; dos hileras de cerdas frontales dirigidas hacia adentro, placa orbital oscura hasta la mitad; parafrontal enteramente recubierto de pruina plateada, mitad anterior sin setas, mitad posterior con dos hileras de setas robustas, casi iguales a las frontales, adicionalmente se observa una tercera hilera de setas cerca de las órbitas, más pequeñas e irregulares; impresión transversa debajo del parafacial café rojizo; sutura fronto-genal con setas cortas casi hasta la base del post-pedicelo; antenas cortas, pedicelo café negruzco, post-pedicelo casi 4 veces la longitud del pedicelo; arista más corta que el post-pedicelo, aristómeros 2 y 3 casi de la misma longitud (Figura 3, K); Palpos cortos, no alcanzan el ángulo vibrisal, café amarillentos, casi del mismo ancho en toda su longitud, 4 setas en el tercio medio del 
margen inferior, 2-3 setas finas en el tercio apical (Figura 3, L).

Tórax. Grisáceo con 5 bandas de pruina blanquecina, la central más notoria y completa, llega hasta el escutelo, las bandas externas cubren el lóbulo post-pronotal, casi toda la notopleura hasta el área supra alar; lóbulo post-pronotal blanco excepto por una pequeña mancha oscura en el medio; anepisterno y anepimeron negros con una pequeña mancha de pruina blanquecina; Postpronoto con 3 setas en línea; Scuto: acrosticales 3: 3, dorsocentrales 3: 4, intraalares 2: 3, post alares 1, supraalares 1: 3. Proesterno setoso. Katepisterno con 4 setas, 3 en una hilera superior y 1 inferior. Escutelo amarillento, 2 pares de discales, 2 pares de basales, 1 par de subapicales y 1 par de apicales divergentes. Alas con venas amarillentas; $\mathrm{R}_{4+5}$ con 2-3 sétulas en la base; celda $\mathrm{r}_{4+5}$ abierta; $\mathrm{M}$ en ángulo recto, poco sinuosa, termina antes del ápice del ala; sección apical de $\mathrm{CuA}_{1}$ casi la mitad de la sección precedente. Calíptra superior amarillenta, caliptra inferior blanca, con algo de amarillo en la base. Escutelo café amarillento con la base gris; un par de apicales, dos pares de discales y tres pares de laterales, todas más fuertes que las apicales, éstas divergentes.

Abdomen. Negro, tergito 2 con 1 par de setas marginales centrales y 1 par de marginales laterales, tergito 3 con 1 par de marginales centrales y 2 pares de marginales laterales, tergito 4 con 2 pares de marginales centrales y 2 pares de marginales laterales, tergito 5 negro con pruina gris, con 1 par de setas discales y 1 grupo irregular de marginales medias.

Material examinado. $1 \hat{\gamma}$, Pumamarca, Cusco, 3.452 m, -1330', -7155', 15-11-2008, L. Paucar; 1ิึ, Huaro, Quispicanchis, Cusco, 3.196 m, -13\%41', -71³8', 07-01-1999, J. Álvarez; $1 \widehat{\jmath}^{\circ}$ Huacarpay, Quispicanchis, Cusco, 3.085 m, -13 $37^{\circ},-71^{\circ} 43^{\prime}$, 16-03-1995, E. Yábar.

Bionomía. La especie Dolichocnephalia puna fue descrita por Townsend con material colectado en La Oroya (Junín, Perú): $75^{\circ} 53^{\prime} \mathrm{O}, 1^{\circ} 31^{\prime}$ 'S y $3.745 \mathrm{msnm}$. Posteriormente fue citada por Cortés \& Hichins (1979) en las localidades de Chapiquiña y Belén (Chile) con coordenadas de $69^{\circ} 31^{\prime} \mathrm{O}-18^{\circ} 23^{\prime} \mathrm{S}$ y $69^{\circ} 31^{\prime} \mathrm{O}-18^{\circ} 29^{\prime} \mathrm{S}$ respectivamente, las mismas que pueden considerarse como el límite sur para la especie. Cortés (1983) cita esta especie para las localidades de Tarapacá y Antofagasta, las mismas que se encuentran en el rango citado. El material estudiado en Cusco ha sido colectado entre 3.085 y $3.452 \mathrm{msnm}$ con un rango latitudinal entre los $13^{\circ} 30^{\prime} \mathrm{S}$ y $13^{\circ} 41^{\prime} \mathrm{S}$, por lo que se considera que la especie se encuentra dentro de su rango habitual.

Hospederos. Desconocidos.

Comentarios. Se reconoce por la mitad anterior de la parafacialia desnuda y sutura fronto-genal con setas cortas casi hasta la base del post-pedicelo. Es el primer registro para el Cusco.

\section{Germariopsis Townsend, 1915}

Especie tipo: Germariopsis andina Townsend (designación original).

Referencias. Townsend, 1915: 66 (descripción género); Townsend, 1941: 30 (redescripción); Guimaraes, 1971: 175 (catálogo).

\section{Diagnosis}

Cabeza dolicocéfala; antenas negruzcas, palpos largos, casi tan largos como el borde genal inferior, amarillos; scutum grisáceo con 5 bandas de pruina blanquecina, la central llega hasta casi el escutelo, se torna amarillenta pasando la sutura; escutelo amarillo con la base oscura, un par de discales, un par de apicales y 3 laterales más largas, celda $\mathrm{r}_{4+5}$ cerrada en el margen del ala y con un corto peciolo; abdomen café oscuro, cerdas marginales, sin discales.

Cabeza. Dolicocéfala, ojos desnudos, oscura en el parafrontal y con pruina plateada en las parafacial; vitta frontal café oscuro, más ensanchada en el triángulo ocelar; placa orbital negra hasta casi la base de las antenas; tres hileras de cerdas frontales, la externa irregular, la cerda frontal más baja casi hasta la base del post-pedicelo, sutura fronto-genal sin setas, parafacial con 2-3 hileras de cerdas casi tan gruesas como las frontales; impresión transversa debajo del parafacial café rojizo; antenas negruzcas, post-pedicelo casi 2,5 veces la longitud del pedicelo, arista desnuda más larga que el post-pedicelo; aristómero 3 casi 1,5 veces la longitud del segundo (Figura 4, A). Palpos largos, casi tan largos como el borde genal inferior, amarillos, ligeramente engrosados apicalmente, 2 setas en el tercio medio del margen inferior, sin setas en el margen superior, tercio apical con un grupo de setas cortas, dispersas (Figura 4, B).

Tórax. Grisáceo con 5 bandas de pruina blanquecina, la central llega hasta casi el escutelo, 

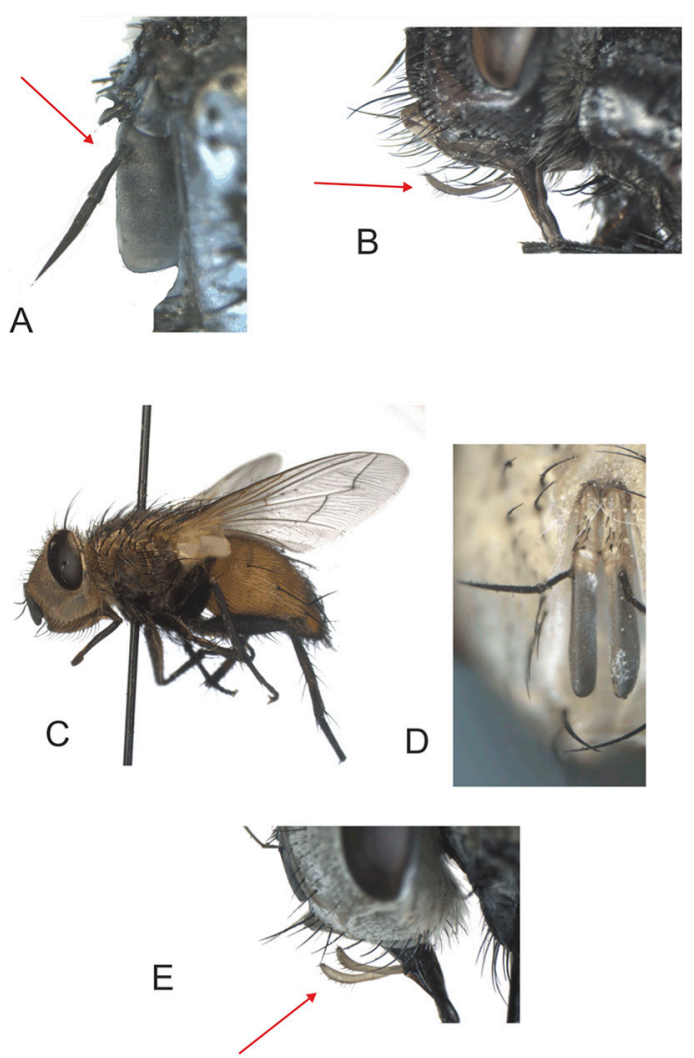

Figura 4. A: Germariopsis, antena; B: Germariopsis, palpo; C: Gonia, hábito; D: Gonia, antena; E: Gonia, palpo.

se torna amarillenta pasando la sutura; una banda blanca que cubre el lóbulo post-pronotal, casi toda la notopleura hasta la base del ala; casi todo el lóbulo post-pronotal blanco excepto por una mancha oscura en el medio; anepisterno negro con una mancha de pruina blanquecina en la mitad posterior. Postpronoto con 3 setas en línea; Scuto: acrosticales 4: 2, dorsocentrales 3: 3, intraalares $1: 3$, post alares 2 , la posterior más desarrollada, supraalares 1: 3. Proesterno setoso. Katepisterno con 4 setas, 3 en una hilera superior, la posterior más desarrollada y 1 inferior. Escutelo amarillento, 2-3 pares de discales, 2 pares de basales, 1 par de subapicales y 1 par de apicales divergentes. Alas con venas amarillentas; celda $\mathrm{r}_{4+5}$ cerrada exactamente en el margen alar y con un peciolo corto; $\mathrm{R}_{4+5}$ con 2 sétulas cortas en la base; $\mathrm{M}$ formando un ángulo agudo y uniéndose a $\mathrm{R}_{4+5}$ antes del ápice del ala; sección apical de $\mathrm{CuA}_{1}$ menos de la mitad de la sección precedente. Calíptras superior amarilla, caliptra inferior blanca con el borde café; escutelo amarillo con la base oscura, un par de discales, un par de apicales y 3 laterales más largas.

Abdomen. Café oscuro sin setas marginales medianas y 1 par de marginales laterales, tergito 3 con 1 par de marginales medianas y 2 pares de marginales laterales, tergito 4 con una hilera de setas marginales separadas entre sí, tergito 5 café oscuro con 1 hilera de marginales medias y varias setas discales.

Material examinado. 1 $\delta^{\lambda}$, Santa Bárbara, Canchis, Cusco, $4.021 \mathrm{~m},-14^{\circ} 09^{\prime},-71^{\circ} 11^{\prime}, 11-8-$ 94, Yábar col.

Bionomía. La especie Germariopsis andina fue descrita por Townsend con material colectado en el cerro San Cristóbal (Lima): $77^{\circ} 01^{\prime} \mathrm{O}, 12^{\circ} 02^{\prime} \mathrm{S}$ y 243.84-365.76 msnm. El rango latitudinal se amplía grandemente ya que el ejemplar estudiado fue colectado a $14^{\circ} \mathrm{S}$, igualmente, su rango altitudinal alcanza una diferencia considerable registrándose actualmente $4.021 \mathrm{msnm}$. A pesar de contarse con un solo ejemplar, la altura en que fue capturado se caracteriza por su ambiente seco, dominado por pastizales y vegetación arbustiva, en esta zona predomina la actividad ganadera y se observan pequeños campos de cultivo, especialmente papa.

Hospederos. Desconocidos.

Comentarios. Se reconoce por la celda $\mathrm{r}_{4+5}$ con un corto peciolo y el abdomen café oscuro sin manchas. Existen algunas pequeñas diferencias con la descripción original: las uñas son bastante más largas que el último segmento tarsal (no más largas en la descripción original); cabeza claramente dolicocéfala (..sólo un poco alargada en el original..); no presenta cilios (cilios de la facialia débiles o incluso vestigiales en el original); frontoclipeo fuertemente producido. Por estas razones mantenemos el ejemplar estudiado en el género Germariopsis con ciertas limitaciones. Es el primer registro para Cusco.

Gonia Meigen, 1803

(Figura 4, C: hábito)

Salmacia Meigen, 1800: 38. Especie tipo: Musca capitata De Geer (nombre suprimido por el Código Internacional de Nomenclatura Zoológica 1963).

Especie tipo: Gonia bimaculata Wiedemann (Sabrosky \& Arnaud, 1965: 1075).

Referencias. Meigen, 1803: 280 (descripción género); Aldrich, 1934: 86 (clave especies); Townsend, 1941: 31 (redescripción); Cortés y 
Campos, 1970: 83 (clave especies); Guimaraes, 1971: 175 (catálogo); Cortés \& Campos, 1974: 113 (clave géneros); Cortés \& Hichins, 1979: 113 (distribución); González y Vergés, 2004: 55 (redescripción).

\section{Diagnosis}

Longitud 10,89 $\pm 0,73(n=40)$; Cuerpo café amarillento, cabeza no alargada; ojos desnudos; antenas amarillentas, palpos largos, alcanzan el ángulo inferior de la gena, engrosados apicalmente, café; scutum con cuatro bandas negruzcas, muy difuminadas y coalescentes dando al mesonoto una tonalidad casi enteramente negruzca hasta antes del escutelo; escutelo amarillo con dos setas apicales pequeñas, un par de discales y tres pares de laterales muy desarrolladas; celda $\mathrm{r}_{4+5}$ cerrada exactamente en el margen alar; abdomen café amarillento con una banda de pruina plateada en los márgenes anteriores de los tergitos 3 y 4 además de una mancha negra central en el margen posterior de estos tergitos; tergito 5 café amarillento.

Cabeza. No alargada, perfil frontal mayor que el perfil facial; sutura fronto-genal sin setas; frontoclipeo poco desarrollado, casi al nivel del borde facial al nivel de la base de las antenas; frente muy inflada; vitta frontal amarilla, del mismo color de la placa orbital, no discernibles entre sí; dos hileras de setas frontales orientadas hacia adentro sin llegar a tocarse, una tercera hilera frontal externa con setas más pequeñas e irregulares; hembra con dos setas fronto orbitales proclinadas; parafacialia más ancha que el diámetro del ojo, amarilla con pruina plateada; cubierta con setas pequeñas y con una hilera anterior de setas más desarrolladas cerca de la sutura fronto-genal; surco genal amarillo, desnudo; sutura fronto-genal sin setas; antenas amarillentas; escapo, pedicelo y base del postpedicelo amarillos, resto del post-pedicelo negro, post-pedicelo casi 3 veces la longitud del pedicelo; arista negra, desnuda, aristómero 3 algo más larga que el aristómero 2, agudo (Figura 4, D); palpos largos, alcanzan el ángulo inferior de la gena, engrosados apicalmente, café, margen inferior con setas finas entre el tercio medio y casi el tercio apical, margen superior sin setas, tercio apical con 5-6 setas muy pequeñas (Figura 4, E).

Tórax. Scutum con cuatro bandas negruzcas, muy difuminadas y coalescentes dando al mesonoto una tonalidad casi enteramente negruzca hasta antes del escutelo; lóbulo post pronotal y notopleura café amarillento; anespiterno y anepimeron café con tonalidad gris; katepisterno café con gris en la mitad inferior; Postpronoto con 3 setas en línea; Scuto: acrosticales 3: 3, Dorsocentrales 3: 4, intraalares 2: 3 , post alares 2, la posterior más desarrollada, supraalares 1: 3 . Proesterno setoso. Katepisterno con 4 setas, 3 en una hilera superior, la posterior más desarrollada y 1 inferior. Escutelo amarillento, 1 par de discales, 1 par de basales, 2 pares de de subapicales y 1 par de apicales cortas y divergentes, setas escutelares marginales muy desarrolladas. Alas con venas amarillentas; celda $\mathrm{r}_{4+5}$ cerrada exactamente en el margen alar; base vena $\mathrm{R}_{4+5}$ con una hilera de sétulas cortas finas entre la base y $1 / 3$ de la distancia entre $R_{4+5}$ y $\mathrm{r}-\mathrm{m}$; $\mathrm{M}$ formando un ángulo agudo, termina antes del ápice alar sin unirse a $\mathrm{R}_{4+5}$, sección apical de $\mathrm{CuA}_{1}$ menos de la mitad de la sección precedente. Calíptras amarillas con el borde café.

Abdomen. Café amarillento con una banda de pruina plateada en los márgenes anteriores de los tergitos 3 y 4 además de una mancha negra central en el margen posterior de estos tergitos; tergito 2 sin setas marginales medianas y 1 par de marginales laterales, tergito 3 con 1 par de setas marginales medianas y 1 par de marginales laterales, tergito 4 con 1 hilera de setas marginales, tergito 5 amarillento con una banda longitudinal central negruzca, varias discales y un grupo se setas marginales más pequeñas

Material examinado. 1옹 Sacsayhuaman, Cusco, 3.580 m, -13³0', -7155', 02-06-1995, E. Yábar; 3ㅇ, Sacsayhuaman, Cusco, 3.580 m, -13³0', -7155', 26-02-1995, E. Yábar; 29 , Kayra, Cusco,


Kayra, Cusco, 3.310m, -13³3', -7152', 08-12-1995, N. Sequeiros; 4, Kayra, Cusco, $-13^{\circ} 33^{\prime},-71^{\circ} 52^{\prime}$, 3.229m, -13 ${ }^{\circ} 33^{\prime},-71^{\circ} 52^{\prime}, 03-01-2004$, W. Cosio; 2 , Huayllojo, Canchis, Cusco, $3.551 \mathrm{~m},-14^{\circ} 01^{\prime}$, -71²6', 18-11-1993, E. Yábar; 2ᄋ, Huayllojo, Canchis, Cusco, $3.551 \mathrm{~m},-14^{\circ} 01^{\prime},-71^{\circ} 26^{\prime}, 03-$ 06-1993, E. Yábar; 4, Huayllojo, Canchis, Cusco, $3.551 \mathrm{~m},-14^{\circ} 01^{\prime},-71^{\circ} 26^{\prime}, 07-12-1994$, E. Yábar; 19, Ollantaytambo, Urubamba, Cusco, $3.025 \mathrm{~m},-13^{\circ} 15^{\prime},-71^{\circ} 26^{\prime}, 20-11-1994$, Y. Valdez; 19, Ollantaytambo, Urubamba, Cusco, $2.470 \mathrm{~m}$, $3.025 \mathrm{~m},-14^{\circ} 01^{\prime},-71^{\circ} 26^{\prime}, 24-12-1994$, Y. Valdez \& A. Alfaro; 29 , Ollantaytambo, Urubamba, Cusco, $3.025 \mathrm{~m},-14^{\circ} 01^{\prime},-71^{\circ} 26^{\prime}, 12-02-2005$, Y. Valdez \& 
A. Alfaro; 29 , Ollantaytambo, Urubamba, Cusco, $3.025 \mathrm{~m},-14^{\circ} 01^{\prime},-71^{\circ} 26^{\prime}, 12-02-2005$, J. Álvarez; 5, Urubamba, Cusco, $2.877 \mathrm{~m}, 2.869 \mathrm{~m},-13^{\circ} 18^{\prime}$, $-72^{\circ} 07$ ', 11-11-2003, A. Alfaro \& Y. Valdez; 1우, Huatata, Urubamba, Cusco, $3.717 \mathrm{~m},-13^{\circ} 24^{\prime},-72^{\circ} 04^{\prime}$, 15-08-2002, E. Yábar; 2ᄋ, Maras, Urubamba, Cusco, 3.375 m, -1320', -72 $09^{\circ}$, 09-02-2008, E. Yábar; 1우 Huito, Canchis, Cusco, 3.360 m, -1400', -71²6', 16-02-1998, E. Yábar; 2ᄋ, Urcos, Quispicanchi, Cusco, 3.136 m, -13\%41', -71'37', 0707-2002, -13 $41^{\circ},-71^{\circ} 37^{\prime}$, A. Alfaro; 1 , , Sicuani, Cusco, $3.672 \mathrm{~m},-14^{\circ} 16^{\prime},-71^{\circ} 13^{\prime}, 07-12-1994$, E. Yábar; 29, Santo Tomás, Cusco, 3.661 m, -14²7', -7204', 19-01-1995, E. Yábar.

Bionomía. En Cusco se han colectado varios ejemplares entre los 3.025 y $3.717 \mathrm{msnm}$. Existe una gran variación cromática y, posiblemente, se trate de diferentes especies. El género presenta un amplio rango geográfico, Avalos (1988) lo cita “... desde la región patagónica argentina por Chile, Brasil, Perú y Ecuador...". Se mencionan varias especies (Cortés \& Hichins, 1979; Cortés, 1983; Avalos, 1988; Vergara \& Raven, 1989; González \& Vergés, 2004) y otras por describir (Cortés, 1983). Es un género particularmente común en las localidades de colección y muy atractivo por su coloración. Los adultos, de actividad diurna, se encuentran volando sobre vegetación natural y cultivos, son muy abundantes especialmente en campos de alfalfa.

Hospederos. Se mencionan varias especies de hospederos: Spodoptera peruviana y S. frugiperda, Agrotis ipsilon, Feltia sp. (Vergara \& Raven, 1989), Agrotis subterranea (Avalos, 1988), Feltia sp., Feltia experta (Cortés \& Hichins, 1979).

Comentario. Es muy difícil separar las posibles especies del género. El género queda bien definido con la descripción de Townsend (1941). El material estudiado traza bien a las descripciones de Phosocephalops fulvus Townsend (1927), Gonia pallens Wiedemann y G. chilensis (Macquart, 1842), Salmacia peruviana Townsend (Townsend, 1912) y G. lineata Macquart (Macquart, 1851), lo que coincide plenamente con lo expresado por Van der Wulp (1903) que sinonimiza G. pallens Wiedemann $=G$. chilensis Macquart $=$ Gonia angusta Macquart y G. lineata Macquart y es incluido en la clave de Van der Wulp (1903).

En la clave de Cortés \& Campos (1970) podrían distinguirse hasta 5 especies de Gonia; en la alternativa 1 se distinguen dos grupos: "abdomen café rojizo sin polen" y "abdomen amarillento, a menudo traslúcido, siempre con polen". En el material examinado no se distinguen estos caracteres y, mientras no se haga un estudio detallado de genitalias y otros caracteres parece mejor ubicar todo el material estudiado como parte del género, sin descartar la posibilidad de que se trate de un complejo de especies.

\section{Discusión}

La taxonomía de Tachinidae, a pesar de los importantes avances logrados, sigue siendo muy difícil, especialmente por el número de géneros que contiene (O'Hara, 2013). Los aspectos biológicos han ayudado a la determinación del complejo de Goniinae (Goniini-Carceliini-Sturmiini-Eryciini) teniendo en cuenta su comportamiento reproductivo, lo cual hace difícil la separación de Goniini y Eryciini con base en caracteres morfológicos (O'Hara, 2013) por lo que se señala que la tribu Goniini se restringe a formas microovíparas mientras que las formas ovíparas y ovolarvíparas son incluidas en la tribu Eryciini (Herting, 1960: original no consultado, citado por O'Hara, 2013: 19). Este aspecto es especialmente importante en el Neotrópico (Toma, 2012).

A pesar de las limitaciones encontradas se ha logrado la identificación de seis géneros, todos ellos citados por primera vez para Cusco, lo cual representa un avance importante en el conocimiento de esta familia. Los taquínidos registrados hasta el momento adquieren mayor importancia si se tiene en cuenta que pueden parasitar diferentes especies de lepidópteros considerados plagas de importancia económica. Asimismo, su comportamiento microovíparo nos hace entender que tienen estrechas relaciones coevolutivas con cultivos y especies vegetales silvestres. Por otra parte, sus rangos, tanto altitudinales como latitudinales, indican que sus especies se encuentran perfectamente adaptadas a las condiciones climáticas de las zonas estudiadas: Provincias Yungas, Prepuna, Atacama, Puna y la Sub-región Chilena central (Morrone, 2017).

Las similitudes entre las áreas citadas han sido resaltadas por Cortés (1983) quien menciona que los cultivos prioritarios para la zona de Tarapacá y Antofagasta son maíz, alfalfa, habas y papas, muy similar a lo encontrado en las zonas de colecta en Cusco. También menciona que la distribución de los taquínidos en Chile se correlaciona fuertemente con la altitud y la vegetación. Adicionalmente se 
estima que la fauna de taquínidos de Tarapacá y Antofagasta tiene mucha afinidad con la región de los Andes occidentales de Perú, Ecuador y Bolivia (Cortés, 1983).

Como se señaló, el estudio de los Tachinidae en Cusco son precarios o inexistentes. Sin embargo, algunos trabajos de Goniini, para la costa central del Perú, han sido publicados: Gonia crassicornis (Fabricius), Lima, Ica; G. lineata Macquart, Lima, Ica; G. pallens Wiedemann, Lima, Ica y $G$. peruviana (Townsend), Lima, Lurín; Protogoniops Townsend, Lima (Vergara \& Raven, 1989); para la Sierra central se mencionan Patelloa similis (Townsend) (Eryciini); Incamyia sp., (Blondeliini), Euphorocera peruviana (Townsend) (Exoristini) y Winthemia sp., (Winthemiini) (Alcalá, 1978).
Como se observa, hasta el momento no han sido citados los géneros registrados en este estudio debido, posiblemente, a que las especies citadas en la bibliografía han sido colectadas mediante la crianza de sus hospederos parasitados y no, como se ha hecho ahora, principalmente mediante la colección de adultos voladores.

En cualquier caso, los resultados logrados representan un aporte importante en el conocimiento de la fauna de taquínidos para el Perú pero, además, resalta el hecho de la investigación colaborativa entre dos instituciones con objetivos comunes. Como ha sido señalado por Toma (2012) es realmente importante contar con colecciones de referencia y éste puede ser el primer paso para lograrlo.

\section{Literatura Citada}

Alcalá, P.

1978. Taquínidos parásitos de Copitarsia turbata Herr-Schaff. en el Valle del Mantaro. Revista Peruana de Entomología, 21: 126.

Aldrich, J.M.

1934. Tachinidae. British Museum (Natural History), Diptera of Patagonia and South Chile, 7(1): 1-170.

Avalos, D.S.

1988. Moscas Tachinidae de la provincial de Córdoba (Argentina). Revista Peruana de Entomología, 31: 48-50.

Cortés, R.

1944. Algunos géneros Goniinos de taquínidos chilenos (Dip., Tachinidae). Agricultura Técnica Chile, 2: 115-124.

Cortés, R.

1983. Tachinid flies (Diptera: Tachinidae) from Tarapacá and Antofagasta provinces, Chile. III. Addendum. Florida Entomologist, 66: 377-389.

Cortés, R.

1986. Taquínidos de Aysén (XI Región) y Magallanes (XII. Región). Chile (Diptera: Tachinidae). Acta Entomológica Chilena, 13: 133-160.

Cortés, R.; Campos, L.

1970. Taquínidos de Tarapacá y Antofagasta. Anales de la Universidad del Norte, 8: 1-104.

Cortés, R.; Campos, L.

1974. Taquínidos de Tarapacá y Antofagasta. Addenda I. (Diptera, Tachinidae). IDESIA, 3: 111-125.

Cortés, R.; Hichins, N.

1979. Taquínidos de Tarapacá y Antofagasta (Diptera: Tachinidae), Addenda II. IDESIA, 5: 111-116.

Cumming, J.M. \& Wood, D.M.

2009. Adult morphology and terminology In Brown, B.V.; Borkent, A.; Cumming, J.M.; Wood, D.M.; Woodley, N.E.; Zumbado M.A. (Eds.). Manual of Central American Diptera Volume 1. NRC Research Press, Ottawa, Ontario, Canada. pp. 9-50.

DeVries, P.J.

1984. Butterflies and Tachinidae: does the parasite always kill its host?. Journal of Natural History, 18: 323-326.
González, C.R.; Vergés, X.

2004. Revisión de las especies de la tribu Goniini de distribución chilena (Diptera: Tachinidae). Acta Entomológica Chilena, 28: 1-24.

Gramajo, M.C.; Diodato, L.; Fuster, A.

2013. Tachinidae (Diptera) de ecosistemas naturales del Chaco semiárido de Santiago del Estero, Argentina. Acta Zoológica Lilloana, 57(2): 212-216.

Guimaraes, J.H.

1971. Family Tachinidae (Larvaevoridae). En: Papavero, N. (ed.). A Catalogue of the Diptera of the Americas South of the United States. Vol. 104. Museu de Zoologia, Universidade de Sao Paulo. Sao Paulo, Brazil. pp. 333.

O'Hara, J.E.

2013. History of tachinid classification (Diptera, Tachinidae). ZooKeys, 316: 1-34.

Meigen, J.W.

1803. Versuch einer neuen Gattungseintheilung der europäischen zweiflügen Insekten. Magazin für Insektenkunde, 2: 259-281.

Morrone, J.J.

2001. Biogeografía de América Latina y el Caribe. vol. 3. M\&T-Manuales \& Tesis SEA, Zaragoza, España, $148 \mathrm{p}$.

Morrone, J.J.

2017. Neotropical biogeography: regionalization and evolution. CRC Press Boca Ratón, USA, 282 p.

O'Hara, J.E.

2013. History of tachinid classification (Diptera, Tachinidae). Zookeys, 316: 1-34.

Sabrosky, C.W.; Arnaud Jr., P.H.

1965. Tachinidae. In: Stone, A.; Sabrosky, C.W.; Wirth, W.W.; Foote, R.H.; Coulson, J.R. A catalog of the Diptera of America north of Mexico, USDA. Agriculture Handbook 276. US. pp. 961-1108.

Stireman, J.O.; O’Hara, J.E.; Wood, D.M.

2006. Tachinidae: evolution, behaviour, and ecology. Annual Review of Entomology, 51: 525-555. 
Toma, R.

2012. Tachinidae, una discusión sobre el problema de la identificación de los taxones de la Región Neotropical. Entomotropica, 27: 145-172.

Townsend, C.H.T.

1912. Descriptions of new genera and species of muscoid flies from the Andean and Pacific Coast regions of South America. Proceedings of the United States National Museum, 43: 301-367.

Townsend, C.H.T.

1915. New Andean Spallanzaniine Flies. Insecutor Inscitiae Menstruus, III: 63-69.

Townsend, C.H.T.

1928. New muscoid genera and species of the coast of Peru and Chile. Revista Chilena de Historia Natural, 31: 158-164.

Townsend, C.H.T.

1936. Part IV. Oestroid classification and habits (Dexiidae and Exoristidae). En: Manual of Myiology. Pri- vately published. Itaquequecetuba, Sao Paulo, Brasil. pp. 169-180.

Townsend, C.H.T.

1941. Part XI. Oestroid Generic Diagnoses and Data. Goniini to Trypherini. En: Manual of Myiology. Privately published. Itaquequecetuba. Sao Paulo, Brasil. Pp. 8-52.

Van der Wulp, F.M.

1903. Biologia Centrali-Americana. Insecta. Diptera, vol. II: 3-250.

Vergara, C. \& Raven, K.G.

1989. Tachinidae registrados en el Museo de Entomología de la Universidad Nacional Agraria La Molina. Revista Peruana de Entomología, 32: 93-101.

Wood, D.M. \& Zumbado, M.A.

2010. Tachinidae, In: Brown, B.V., A. Borkent, J.M. Cumming, D.M. Wood, N.E. Woodley \& M.A. Zumbado (Eds.). Manual of Central American Diptera. Vol. 2. CNRC Research Press. Ottawa, Canada. pp. 1343-1417. 